\title{
Wrist Kinematics from Computed Tomography Data
}

\author{
Maarten Beek, Carolyn F. Small, Steve Csongvay, Rick W. Sellens, R.E. Ellis, and
} David R. Pichora

\author{
Human Mobility Research Centre \\ Kingston General Hospital \\ Kingston, ON, K7L 2V7 \\ Canada \\ beek@me.queensu.ca
}

\begin{abstract}
Three-dimensional (3-d) surface models of human carpal bones obtained from Computed Tomography (CT) were used to investigate their kinematic behavior throughout the entire physiological range of motion of the human wrist joint. The 3-d motion of the bones was visualized graphically by the finite helical axis (FHA) and smooth animations. It was found that extension mainly occurs in the radial-carpal joint and flexion is shared between the radialcarpal and midcarpal joints. During radial and ulnar deviation, the relative motion between the scaphoid and lunate was larger than in flexion-extension. This study will improve our understanding of carpal bone motion in a range of wrist poses, and will provide morphological data for the design of a functional wrist replacement arthroplasty.
\end{abstract}

\section{Introduction}

A survey conducted in 2003, showed that arthritis costs the Canadian economy CAN\$ 4.4 billion each year. The wrist joint is often involved and treatment options are limited. They include carpectomy, limited fusion or complete fusion. Wrist replacement is seldom performed due to the high failure rates of the presently available prostheses. An increased knowledge of the kinematics of the joint will assist in the design process of more successful arthroplasties.

\section{Materials and Methods}

Four fresh frozen human upper limbs were scanned in 11 different poses throughout their entire range of motion. Surface models of the carpal bones were created from the obtained CT images. Due to the small size and proximity of carpal bones, manual segmentation of the CT images was necessary. The Iterative Closest Point algorithm was applied to register the models of different poses to each other. The kinematics of each bone was described by a FHA [1]. Smooth animations of wrist motion were created to verify the kinematics visually ${ }^{1}$. The surface models were compared with point clouds generated by a laser scanner. The intra- and inter-operator variability was determined by comparing the surface areas and volumes of the models.

\footnotetext{
${ }^{1} \mathrm{http}: / /$ me.queensu.ca/hmrc/research/KAJ_movie.html 


\section{Results}

The mean error between surface models and point clouds was less than $0.1 \mathrm{~mm}(\mathrm{n}=9)$. The surface areas and volumes of the models were in agreement with other studies [2]. The kinematic analyses showed that extension mainly occurred in the radiocarpal joint and that flexion was shared between the radiocarpal and midcarpal joints. Compared with flexion-extension, the relative motion between the scaphoid and lunate was larger in radial-ulnar deviation, when the scaphoid moved out of the plane of motion. In pronation-supination motion, the inter-carpal motion was minimal.

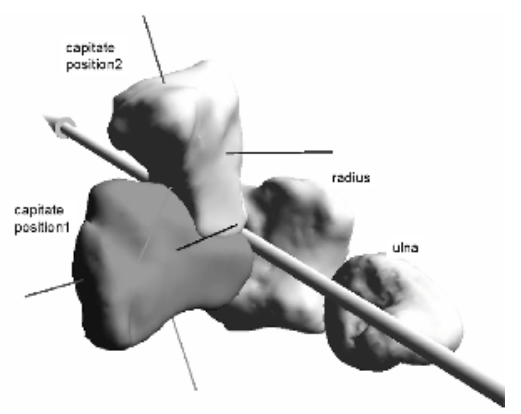

Fig. 1. The finite helical axis (arrow) describing the 3-d motion of the capitate bone when the wrist moves from neutral (position1) to $65^{\circ}$ flexion (position2). The principal axes, shown in the models of the bone in both poses as lines, were used to determine its motion. The distal parts of the radius and the ulna are shown for reference

\section{Discussion}

Surface models obtained from CT images, were successfully applied to determine the kinematics of human wrists. Data from healthy and arthritic living subjects leading to more physiologically realistic results will be gathered once protocols and software tools are developed and validated. The created surface models can be applied in additional studies, like Finite Element analyses. Parts of the software can be used for presurgery planning, inter-operative guidance, and post-surgery evaluation.

\section{References}

1. Spoor CW, Veldpaus FE: Rigid body motion calculated from spatial co-ordinates of markers. Journal of Biomechanics 13(4), 391-393, 1980

2. Belsole RJ, Hilbelink DR, Llewellyn JA, Stenzler S, Greene TL, Dale M: Mathematical analysis of computed carpal models. Journal of Orthopaedic Research 6, 116-122, 1988 